\title{
Reference materials and duty of ReCCS
}

\author{
Hirohito Umemoto* \\ Reference Material Institute for Clinical Chemistry Standards (ReCCS), Japan
}

\section{Definition of reference materials}

A reference material is a substance which calibration of a measuring device, evaluation of a measuring method, or one or more characteristic values (concentration) for use in assigning a value are appropriately determined.

A certified reference material is a reference material which one or more characteristic values are provided by metrological relevant procedure with a certificate where characteristic values, their uncertainties and metrological traceability are described.

The reference materials can be divided into two classifications. One is a pure substance system reference material, called primary reference material, the other is a standard material having the same composition as the actual sample for example serum and blood. In other words, it is a standard material of biological origin, which is required especially in the field of clinical examination, hereafter named a biological matrixbased certified reference material.

Usually, primary reference materials are often used as national measurement standards. In the field of clinical examination which requires easy-to-use approach, quickness and precision, biological matrix-based certified reference materials are preferred. When a biological matrix-based certified reference material is used, a traceability can be secured in the case of the existence of a standard measurement method that connects the biological matrix-based certified reference material and the primary reference material. This is the reason why the biological matrix-based certified reference materials are used for the accuracy management of the routine test methods.

In the field of clinical examination, from the highest in the traceability system, reference materials are distinguished as follows: primary reference materials (including national measurement standard reference materials, etc.), reference measurement standards, working measurement standards, calibrators, or controls. Reference measurement standard materials are used as a direct criterion of the of the routine method's accuracy. The reference measurement standard should be the same as the samples used for actual measurement.

\section{Duty of ReCCS}

\section{Internationally certified reference laboratory in laboratory} medicine

ReCCS (Reference Material Institute for Clinical Chemistry Standards), is certified as a reference laboratory in the list of JCTLM (Joint Committee on Traceability in Laboratory Medicine) regarding cholesterol, HbA1c, etc., and plays a duty of primary reference laboratory in Laboratory Medicine in Japan.

\section{Supplying institute of reference materials}

ReCCS is providing reference materials by the international standards applied by MRA (Mutual Recognition Agreement), such as Certified Reference Material for Measurement of HbA1c (JCCRM 411), HDL and LDL Cholesterol (JCCRM 224), Total Cholesterol (JCCRM 211), and Creatinine (JCCRM 521).

As higher-order reference materials registered by JCTLM (The Joint Committee for Traceability in Laboratory Medicine), ReCCS is providing the Certified Reference Materials for HbA1c (JDS HbA1c Lot 2), Triglycerides (JCCRM 223), Cholesterol (JCCRM 211), Electrolytes (JCCRM111).

ReCCS has obtained three major ISO certifications for the first time in Japan, ISO 15195, ISO/IEC 17025 and ISO Guide 34, for lipids which include Total Cholesterol, Total Glycerides, HDL cholesterol and LDL cholesterol, as well as $\mathrm{HbA1c}$ and Creatinine.

ReCCS is an organization who certifies and provide the reference materials for using in the field of clinical examination such as primary measurement standards, reference measurement standards and biological matrix-based certified reference materials.

\section{Role to traceability system}

As most of the reference measurement standards of ReCCS are based on biological samples their certified values are not easily affected even in the presence of coexistence of other ingredients, and these certified values are measured using isotope dilution mass spectrometry (ID/ MS). Some of the reference measurement standards are determined by other reference methods, but these are methods, certified as reference methods by the academic societies in Japan.

Also, as for the reference measurement standard is most important to secure the traceability system, we are checking the bias between several routine methods.

\section{Collaboration with academic organizations and institutions both internationally and domestically}

In recent years, ensuring the traceability of the values, measured by certified reference materials has become indispensable because of the expanding acquisition of ISO 15189, rapid progress of international standardization, and securing the reliability of the laboratory test results.

${ }^{\star}$ Correspondence to: Hirohito Umemoto, Reference Material Institute for Clinical Chemistry Standards (ReCCS), Azaminominami, Aoba-ku, Yokohama, Kanagawa 225-0012, Japan, E-mail: mumemoto@reccs.net

Received: February 20, 2019; Accepted: February 25, 2019; Published: February 27, 2019 
Japanese Association of Medical Technologists has proceeded accuracy management projects of the laboratory test results using certified standard reference materials which have been provided from ReCCS. (Also reference materials of IRMM have been used.)

The biological matrix-based primary reference materials of ReCCS have acquired MRA and their values are internationally acceptable. Manufacturers that provide reference materials in new fields are increasing both internationally and domestically.

The importance of international reference materials, which have acquired MRA, is increasing because the consistency with values of the overseas manufacturers' reference materials is indispensable.

Ministry of Health, Labor and Welfare / In vitro diagnostic standards for calibration In order to confirm performance of in vitro diagnostic standards according to the standards of in vitro diagnostic medicine by the Minister of Health, Labor and Welfare by the Article 41, Paragraph 3 of the Pharmaceuticals Affairs Law related to ensure the quality, effectiveness and safety, ReCCS is supplying certified standard reference materials. (It is said that when the performance of the in vitro diagnostic medicine products depends on the use of the calibrator or the reference materials, retroactivity of values which was assigned to them must be guaranteed using available standard measuring methods or higher-order reference materials.)

\section{Features of reference materials of ReCCS}

Reference Materials of ReCCS are to be used for the purposes described below: a. Calibration of routine method

b. Evaluation of precision of routine method (manufacturer for in vitro diagnostic medicine products and medical institutions)

c. Criteria for determining the calibrator and control values of the routine method

d. Evaluation of accuracy of new developed and improved routine method

e. Calibration materials for manufacturing and sales of in vitro diagnostic medicine products to the Ministry of Health, Labor and Welfare

f. Criteria of target values for internal quality control and external quality control

g. Securing traceability by ISO 15189

\section{Main standard substance provided by ReCCS Glycated Hemoglobin Glucose}

Cholesterol (Total cholesterol, HDL cholesterol, LDL cholesterol), Triglycerides, Total Glycerides, Free Glycerol, Electrolytes (Na, K, Cl, total $\mathrm{Ca}$, total $\mathrm{Mg}$ ), Urea nitrogen, uric acid, Serum iron, Inorganic phosphorus

Total haemoglobin, CRP, Glycated albumin, Albumin, Blood gas, Urine components, JSCC enzymes (Multi-Enzymes, Cholinesterase), IFCC standards, Dialysis Solutions, etc. 